Relations industrielles

Industrial Relations

\title{
The Practice of Industrial Relations, by D. A. Peach and D. Kuechle, Toronto, Mc-Graw Hill Ryerson Ltd., 1975, 358 pp.
}

\section{Rodrigue Blouin}

Volume 33, numéro 1, 1978

URI : https://id.erudit.org/iderudit/028856ar

DOI : https://doi.org/10.7202/028856ar

Aller au sommaire du numéro

Éditeur(s)

Département des relations industrielles de l'Université Laval

ISSN

0034-379X (imprimé)

1703-8138 (numérique)

Découvrir la revue

Citer ce compte rendu

Blouin, R. (1978). Compte rendu de [The Practice of Industrial Relations, by D. A. Peach and D. Kuechle, Toronto, Mc-Graw Hill Ryerson Ltd., 1975, 358 pp.]

Relations industrielles / Industrial Relations, 33(1), 159-160.

https://doi.org/10.7202/028856ar

Tous droits réservés @ C Département des relations industrielles de l'Université Laval, 1978
Ce document est protégé par la loi sur le droit d'auteur. L'utilisation des services d'Érudit (y compris la reproduction) est assujettie à sa politique d'utilisation que vous pouvez consulter en ligne.

https://apropos.erudit.org/fr/usagers/politique-dutilisation/ 
de l'arbitrage des griefs et de la Commission des relations du travail. $\AA$ noter ici aussi l'insistance de monsieur Baigont pour bien situer la «supervisory jurisdiction» de la Commission: «(...) when an arbitration award is arrived at in a manner inconsistent with the procedure and principles mandated by the code, then this Board will not hesitate to insinuate itself into the arbitration process. When that mandate has been carried out then the fact that this board does not agree with the arbitrator's interpretation of the collective agreement, is no grounds for a reversal under section 108 of the Code".

En définitive, "Grievance Arbitration: A Review of Current Problems", est un ouvrage qui ne peut être ignoré par aucun intéressé à l'arbitrage des griefs. Pour le lecteur québécois, il apparaîtra certes que les solutions qui y sont rapportées ne sont pas toutes applicables ici, ni même importantes en totalité. Néanmoins, la majorité de ces articles aidera notre doctrine et notre jurisprudence si ces textes sont utilisés avec circonspection. À ce sujet, mentionnons que l'utilisation irréfléchie de solutions adoptées dans un contexte socioéconomique bien particularié et en égard de conventions collectives qui traduisent l'état d'évolution de ce contexte socio-économique, ne pourrait que créer des difficultés de fonctionnement dans notre propre système de relations du travail.

Rodrigue BLOUIN

Université Laval

The Practice of Industrial Relations, by D. A. Peach and D. Kuechle, Toronto, Mc-Graw-Hill Ryerson Ltd., 1975, $358 \mathrm{pp}$.

Ce volume ne peut être considéré comme un exposé systématique du système des relations industrielles. L'ouvrage entend donner un enseignement de la pratique des relations du travail au Canada et aux États-Unis et se veut avant tout destiné au personnel de la gérance et aux étudiants en sciences administratives.

La méthode utilisée par les auteurs est descriptive et généralement complétée par des cas. Dix thèmes, axés exclusivement sur le processus de la négociation collective, ses éléments et ses résultats, sont présentés. Au tout début, on retrouve une introduction de circonstance (thème 1) et une traditionnelle histoire des relations du travail (thème 2). Une présentation de la législation constitue l'objet du troisième thème: cette présentation attache une énorme importance à l'influence américaine. Le quatrième thème, intitulé «Union Organisation", est en fait consacré aux principes de base gouvernant l'accréditation et la négociation entre les parties. Suivent des thèses relatant la théorie et la pratique de la négociation collective (thème 5) et de la médiation (thème 6). Le septième thème porte sur la convention collective, qualifiée de "contract administration", tandis que le huitième thème décrit l'arbitrage des griefs. Le syndicalisme dans la fonction publique fait l'objet du neuvième thème. Enfin, le dernier thème entend aider à la mise en place d'un système valable de relations du travail dans une entreprise.

De façon générale, cet ouvrage se lit bien. Par contre, celui qui a déjà une compréhension de base du système des relations industrielles tirera plus de profit de la lecture que le débutant. Deux observations d'ordre général nous apparaissent nécessaires à ce sujet. En premier lieu, nous estimons que le "système des relations industrielles » ne peut être réduit aux seules relations du travail. Les relations industrielles se préoccupent par exemple, selon notre entendement, des problèmes de la main-d'œuvre (marché du travail, emploi, etc.). De plus, les relations collectives ne forment pas la matière exclusive du champ des préoccupations. On ne peut ignorer par exemple l'apport des normes étatiques du travail. Or, dans le volume, ces diverses considérations sont presqu'ignorées. Ainsi, le débutant risque de percevoir les relations industrielles d'une façon limitative. Il aurait convenu, selon notre opinion, que l'on fasse mention des grandes théories des diverses approches et des auteurs éminents qui ont façonné les relations industrielles. Par ailleurs, il aurait été préférable que certaines considérations qui nous semblent étrangères au système des relations industrielles, soient ignorées. À titre d'exemple, 
nous signalons ce passage: «He openly supported René Lévesque, leader of the Parti québécois, the Québec separatist party, and gave Lévesque $\$(.$.$) of his own$ money to publish a separatist oriented manifesto in 1967. His challengers claimed had channeled CNTU funds into radical political causes and that, himself had used union (...) excessively». En second lieu, il nous a semblé qu'il y aurait eu lieu de dégager d'une façon plus approfondie les dénominateurs communs et les points de différentiation des coordonnées juridiques, institutionnelles et socio-économiques des diverses provinces, quitte à atténuer l'importance démesurée accordée à l'influence américaine.

Rodrigue BLOUIN

Université Laval

New Directions for Manpower Policy, par Barbara Goldman, Montréal C. D Howe Research Institute, 1976, 113 pp.

Cet ouvrage vise essentiellement à présenter, analyser et critiquer ces instruments utilisés par le gouvernement fédéral dans le but d'améliorer le fonctionnement des marchés du travail et regroupés sous le vocable de politique de main-d'œuvre.

L'auteur, pour atteindre son objectif, présente d'abord les objectifs de la politique de main-d'œuvre canadienne et tente de résumer les principaux problèmes de marché du travail au Canada. Puis l'auteur attaque successivement la formation professionnelle comme mesure d'intervention au niveau de l'offre, la création d'emploi comme mesure d'intervention au niveau de la demande, et l'expérience des CMC comme mesure d'appariement sur les marchés du travail. Finalement, à partir de l'analyse effectuée, l'auteur présente une certaine prospective dans le domaine de la main-d'œuvre au Canada.

Il faut admettre comme jugement global, que l'ouvrage de Made Goldman est bien fait et représente un outil de travail essentiel à celui qui s'intéresse à l'aspect maind'œuvre du marché du travail. Cependant, il ne faudra pas y chercher un caractère global et général puisque plusieurs aspects de la question de la main-d'œuvre y ont été volontairement et involontairement laissés de côté. En fait, l'auteur se borne à ce qu'il a été traditionnellement conçu comme essentiel. Sur ce point, encore une fois, ce livre est intéressant. Cependant, il évite des points vitaux de la politique des services de main-d'œuvre.

D'abord, ce n'est pas cet ouvrage qui nous aidera à replacer les efforts de maind'œuvre au Canada dans une perspective historique. Cet aspect est d'autant plus important qu'il nous aidera à comprendre plusieurs des caractéristiques actuelles du système canadien de main-d'œuvre qui existent très souvent simplement parce qu'ils ont été instaurés dans des circonstances particulières par des gens identifiables pour "solutionner" des problèmes particuliers. Un tel aspect historique est d'autant plus important à examiner que le (ou les) programme (s) existe(nt) encore malgré le fait que les circonstances, les hommes et les problèmes ont profondément changé.

Ensuite, l'auteur admet (p. 12) ne se borner, à toute fin pratique, qu'aux principaux programmes qui ont traditionnellement influencé les fonctions essentielles de la politique des services de main-d'œuvre. En fait, l'auteur n'examine que l'aspect qualitatif de l'offre de travail en n'examinant que la formation professionnelle, laissant systématiquement de côté l'aspect quantitatif ( «immigration » contrôle quantitatif, et même l'aspect population à plus long terme) de cette offre de travail. Même si une telle approche est en tout point conforme à l'approche traditionnelle, un effort de «renouveau» aurait été grandement utile sur ce point.

Quant à la formation professionnelle, l'approche utilisée est à la fois conservatrice et "prudemment" novatrice. En effet, l'auteur semble se plier dans un premier temps aux objectifs traditionnels de la formation professionnelle (croissance économique, stabilisation et réduction des disparités de revenus) pour ensuite examiner d'une façon très diplomatique l'expérience d'évaluation de tels programmes qui ne se borne malheureusement qu'à des 